\title{
THEO CHEM
}

\section{Reaction channels of the potential energy surface: application of Newton trajectories}

\author{
Michael Hirsch, Wolfgang Quapp ${ }^{*, 1}$ \\ Mathematical Institute, University of Leipzig, Augustus-Platz, D-04109 Leipzig, Germany
}

Received 27 April 2004; revised 26 May 2004; accepted 3 June 2004

Available online 6 August 2004

\begin{abstract}
The reaction path is an important concept of theoretical chemistry. We employ the definition of the Newton trajectory (NT). An NT follows a curve where the gradient is always a pointer to a fixed direction. Usually, a whole family of NTs connects two adjacent stationary points of an index difference of one. We will name such a family a reaction channel. The border between two reaction channels is formed by singular NTs which cross valley-ridge inflection (VRI) points. Examples are given with the Müller-Brown potential, and the potential energy surfaces of water and formaldehyde.

(C) 2004 Elsevier B.V. All rights reserved.
\end{abstract}

Keywords: Potential energy surface; Reaction path; Projected gradient; Newton trajectory; Reaction channel

\section{Introduction}

The concept of the minimum energy path (MEP) or reaction path (RP) of an adiabatic potential energy surface (PES) is the usual approach to the theoretical kinetics of larger chemical systems [1,2]. It is roughly defined as a line in coordinate space, which connects two minima by passing the saddle point (SP), the transition structure of a PES. The energy of the SP is assumed to be the highest value tracing along the RP. It is the minimal energy a reaction needs to take place.

Reaction theories are based either implicitly (transition state theory), or explicitly (variational transition state theory) on the knowledge of the RP [2]. These theories require local information about the PES along the RP only. They circumvent the dimension problem for medium-sized or large molecules: it is impossible to fully calculate their PES.

The starting point is a geometrically defined pathway which may serve as an RP. Geometrically defined means that only properties of the PES are taken into account, and that no dynamic behavior of the molecule is taken into consideration. Any parameterization $s$ of the $\operatorname{RP} \mathbf{x}(s)=\left(x^{1}(s), \ldots, x^{n}(s)\right)^{T}$ is

\footnotetext{
* Corresponding author. Tel.: +49-341-9732153; fax: +49-3419732199.

E-mail address: quapp@rz.uni-leipzig.de (W. Quapp).

1 www.mathematik.uni-leipzig.de/MI/quapp.
}

called reaction coordinate. We use here the distinguished or driven coordinate method $[3,4]$ in the modern form of RGF [5,6], also called Newton trajectory (NT).

Usually, in one's imagination the MEP is situated in a valley of the PES. But how the RP ascends to the SP is an uncertainty of the general RP definition. That opens the possibility to use a full family of similar trajectories to define a reaction channel: it may be formed by 'all' lines of a special character which connect, for example, the reactant minimum with one SP of interest.

In this article, we use the definition of Newton trajectories (NT) [5,6] to define reaction channels [7]. The older definition of an RP by gradient descent, the intrinsic reaction coordinate (IRC) of Fukui [8], or more general the steepest descent (SD), the 'meta-IRC' [9], opens the possibility to divide the configuration space into basins of attraction, or catchment basins $[1,10]$. They are defined as the set of points that will flow to it through gradient descent. The reaction channels of the NTs defined in this article are another classification scheme for the configuration space. Thus, NTs are curves with an alternate property, in comparison to SD curves.

Since there are different SPs around a minimum, different reaction channels have to exist. The question emerges, what are the borders between the channels? The answer is: every border is formed by NTs leading to valley-ridge inflection (VRI) points, so-called singular NTs. 
The article is organized as follows. Section 2 repeats known properties of a PES and its valley-ridge inflection (VRI) points. With Section 3 the main part begins, it gives some properties of Newton trajectories known up to date. Section 4 extends the study of extraneous singularities like VRIs. Section 5 develops the definition of reaction channels of a PES and the relation of NTs to that important property. It finishes with some examples. In Section 6 we give some conclusions. Appendix A contains the used formulas of the model PESs.

\section{Potential energy surface}

The adiabatic PES of the molecular system of observation is the basis of our treatment. Using the BornOppenheimer approximation, we assume that the movements of the electrons and of the atom kernels are decoupled. The PES is the sum of the Coulomb-repulsion of the atom kernels and the Schrödinger equation of the electrons $\mathscr{H} \Psi=E \Psi$. The explicit calculation of the energy $E$ is not of interest, here. We assume the PES is given by a scalar function of the coordinates of the molecule at every point of interest:

Definition 1. Let $\mathscr{K}$ be an open subset of $\mathbb{R}^{n}$, and let $\mathbf{x}=\left(x_{1}, \ldots, x_{n}\right) \in \mathscr{K}$. The function $E(\mathbf{x})$ with $\mathscr{K} \rightarrow \mathbb{R}$ is an $n$-dimensional potential energy surface (PES). $\mathscr{K}$ is the configuration space of the PES. The derivative $G: \mathscr{K} \rightarrow \mathbb{R}^{n}$ with

$G(\mathbf{x})=\mathrm{d} E(\mathbf{x})=\left(\frac{\partial E}{\partial x_{1}}(\mathbf{x}), \ldots, \frac{\partial E}{\partial x_{n}}(\mathbf{x})\right)^{T}$

is the gradient, and the Hessian matrix $H(\mathbf{x}) \in \mathbb{R}^{n \times n}$ is

$H(\mathbf{x})=d^{2} E(\mathbf{x})=\left(\frac{\partial^{2} E}{\partial x_{i} \partial x_{j}}(\mathbf{x})\right)_{i, j=1}^{n}$

The configuration space of a molecule is restricted. We assume at least a twofold differentiability of the PES for practical reasons-for the use of the diverse applications.

Definition 2. A point $\mathbf{x} \in \mathscr{K}$ is nondegenerate if $\operatorname{det} H(\mathbf{x}) \neq 0$. On the contrary case it is degenerate. The index of a nondegenerate point $\mathbf{x} \in \mathscr{K}$ is the number of negative eigenvalues of $H(\mathbf{x})$. We write ind(x), as well as $\operatorname{ind}_{2}(\mathbf{x}):=\operatorname{ind}(\mathbf{x})(\bmod 2)$.

Modulo 2 means the remainder left when ind(x) is divided by 2 . The value $i n d_{2}(\mathbf{x})$ is equivalent to the sign of the determinant of the Hessian

$$
\begin{aligned}
& \operatorname{det} H(\mathbf{x})>0 \Leftrightarrow \operatorname{ind}_{2}(\mathbf{x})=0 \text { and } \\
& \operatorname{det} H(\mathbf{x})<0 \Leftrightarrow \operatorname{ind}_{2}(\mathbf{x})=1
\end{aligned}
$$

Definition 3. A point $\mathbf{x}_{0} \in \mathscr{K}$ with $G\left(\mathbf{x}_{0}\right)=0$ is named stationary point $(\mathrm{StP})$. $\operatorname{Ess}(\mathscr{K})$ is the set of all stationary points in $\mathscr{K}$. A nondegenerate stationary point, $\mathbf{x}_{0}$, is:

- $\operatorname{minimum}$ if $\operatorname{ind}\left(\mathbf{x}_{0}\right)=0$, or

- $\operatorname{maximum}$ if $\operatorname{ind}\left(\mathbf{x}_{0}\right)=n$, or

- saddle point of index $i$ if $\operatorname{ind}\left(\mathbf{x}_{0}\right)=i, 0<i<n$.

We assume that no stationary point is degenerate, i.e. that for all $\mathbf{x} \in \mathscr{K}$ it holds the regularity condition

$\|G(\mathbf{x})\|+|\operatorname{det} H(\mathbf{x})|>0$.

This convention will frequently occur in the sequel. Nondegenerate stationary points are isolated [11]. A special subset of degenerate points can be interpreted to be the branching points of RPs.

Definition 4. A valley-ridge-inflection point (VRI) is located where the gradient is orthogonal to a zero eigenvector of the Hessian [12]. The subset of such points is $\operatorname{Ext}(\mathscr{K})$.

At a VRI, the gradient does not lie in the kernel of the Hessian, and an augmented Hessian with gradient does not lift the defect of the rank

$\operatorname{Ext}(\mathscr{K})=\{\mathbf{x} \in \mathscr{K} \operatorname{rank}[H(\mathbf{x}), G(\mathbf{x})]<n\}$.

The bracket means matrix augmentation: $[H(\mathbf{x}), G(\mathbf{x})] \in$ $\mathbb{R}^{n \times(n+1)}$. Note that a VRI point needs not be symmetric [13]. VRI points are independent of any curve definition.

\subsection{Projection operator}

It is $S^{n-1}=\left\{\mathbf{x} \in \mathbb{R}^{n}\|\mid \mathbf{x}\|=1\right\}$ the unit sphere in $\mathbb{R}^{n}$. Elements from $S^{1}$ - the unit circle-are given as angles with point $(1,0) \in S^{1}$ to be $0^{\circ}$.

We choose a column vector $\mathbf{r} \in S^{n-1}$ for a projection. It is a unit vector. The transposed vector $\mathbf{r}^{T}$ is a row vector. The dimension of $\mathbf{r}$ is $(n \times 1)$ where that of $\mathbf{r}^{T}$ is $(1 \times n)$. We form the dyadic product $\mathscr{D}_{r}=\mathbf{r} \cdot \mathbf{r}^{T}$ which is an $(n \times n)$ matrix. $\mathscr{D}_{r}$ projects with $\mathbf{r}$

$D_{r} \mathbf{r}=\left(\mathbf{r} \cdot \mathbf{r}^{T}\right) \cdot \mathbf{r}=\mathbf{r}\left(\mathbf{r}^{T} \cdot \mathbf{r}\right)=\mathbf{r}$

where we use the unit length of $\mathbf{r}$ in the scalar product. The projector which projects orthogonally to $\mathbf{r}$ is with the unit matrix I

$\mathscr{P}_{r}=\mathbf{I}-\mathscr{D}_{r}$.

\section{RGF, Newton trajectories}

It was proposed to choose a driving coordinate along a valley, to go a step in this direction, and to perform an energy optimization of the residual coordinates [3]. 
Recently, the method was transformed into a new mathematical form to RGF $[5,6,14]$. The concept is that a selected gradient direction is fixed along the curve $\mathbf{x}(s)$

$G(\mathbf{x}(s)) /\|G(\mathbf{x}(s))\|=\mathbf{r}$,

where $\mathbf{r}$ is the unit vector of the search direction. The search direction may correspond to an assumed start direction of a chemical reaction. Or, it may be the direction between the two minima of reactant and product, or the direction between the reactant and the assumed SP. The property (6) is realizable by a projection of the gradient employing $\mathscr{P}_{r}$ of Eq. (5). We pose

$\mathscr{P}_{r} G(\mathbf{x}(s))=0$.

$\mathscr{P}_{r}$ is a constant matrix of rank $n-1$. It is the kernel $\operatorname{ker}\left(\mathscr{P}_{r}\right)=\operatorname{lin}\{r\}$, the linear space of $\mathbf{r}$, and it is the image $\operatorname{Im}\left(\mathscr{P}_{r}\right)=\operatorname{lin}\{\mathbf{r}\}^{\perp}$, the orthogonal space on $\mathbf{r} . \mathscr{P}_{r}$ projects in direction of $\operatorname{lin}\{\mathbf{r}\}^{\perp}$.

Definition 5. The map $\mathscr{R}: \mathbb{R}^{n} \times S^{n-1} \rightarrow \mathbb{R}^{n-1}$, with $\mathscr{R}(\mathbf{x}, \mathbf{r})=\mathscr{P}_{r} G(\mathbf{x})$ will be called the reduced gradient, and $\mathbf{r} \in S^{n-1}$ will be called search direction. The equation

$\mathscr{R}(\mathbf{x}, \bar{r})=0$

is for any fixed $\overline{\mathbf{r}} \in S^{n-1}$ the reduced gradient equation to the search direction $\overline{\mathbf{r}}$.

On $\mathscr{K} \backslash \operatorname{Ess}(\mathscr{K})$ a differentiable map $\underline{r}: \mathbb{R}^{n} \rightarrow S^{n-1}$, $\underline{r}(\mathbf{x}):=G(\mathbf{x}) /\|G(\mathbf{x})\|$ exists, with $\mathscr{R}(\mathbf{x}, \underline{r}(\mathbf{x}))=0$.

Definition 6. The map $\underline{r}$ is named trajectory map [15]

Based on the explicit definition, the predictor-corrector method of the reduced gradient following (RGF) [6] traces a curve (Eq. (7)) along its tangential vector by the derivative to obtain the tangent $\mathbf{x}^{\prime}$

$0=\frac{d}{\mathrm{~d} s}\left[\mathscr{P}_{r} G(\mathbf{x}(s))\right]=\mathscr{P}_{r} \frac{\mathrm{d} G(\mathbf{x}(s))}{\mathrm{d} s}=\mathscr{P}_{r} \mathbf{H}(\mathbf{x}(s)) \mathbf{x}^{\prime}(s)$.

The RGF is a simple but effective procedure in order to determine all types of StPs [5]. In the general good-natured case, each RGF curve passes each StP. A whole family of RGF curves connects the extrema if we vary the search direction $\mathbf{r}$ [16], see Fig. 1.

Definition 7. Let $r \in S^{n-1}$. We will name Newton trajectory (NT) in $\mathscr{K}$ to the direction $\mathbf{r}$ the set

$\mathscr{T}_{r}(\mathscr{K}):=\{\mathbf{x} \in \mathscr{K} \mid G(\mathbf{x})=r\|G(\mathbf{x})\|\}$.

It is clear that $\mathscr{T}_{r}$ is the set of solutions of Eq.(8). Or, in other words, it fulfills Eq.(6): the gradient points into the same direction. For every nonstationary point $\mathbf{x} \in \mathscr{K} \backslash \operatorname{Ess}(\mathscr{K})$ the $\mathrm{NT}$ is given by the direction of the gradient. The name NT is coming from another defining equation of this kind of curves, the so-called

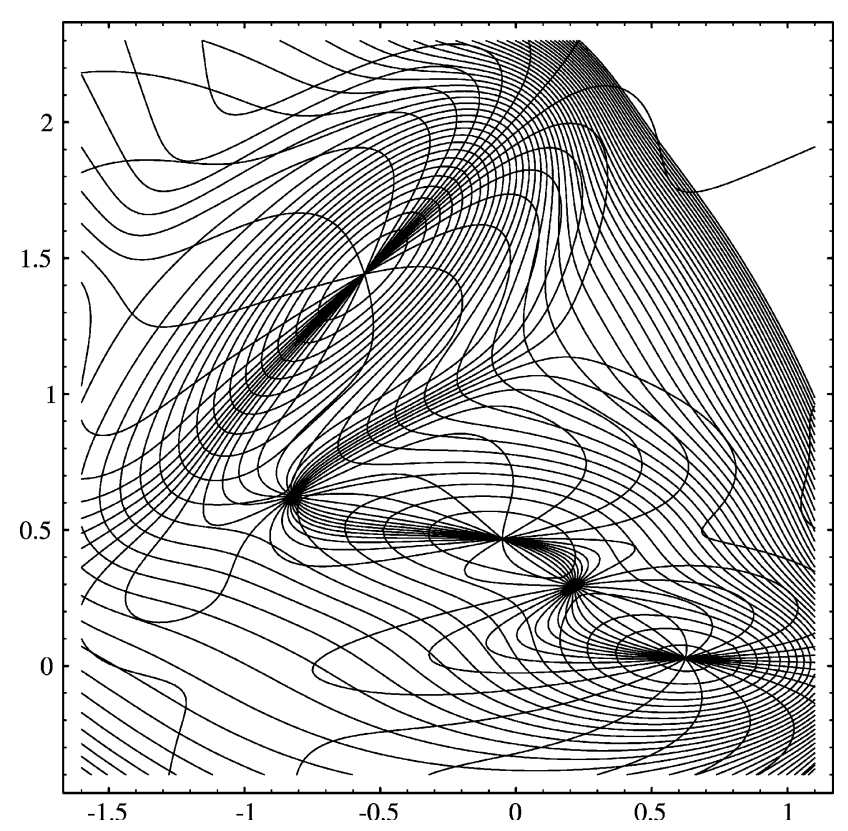

Fig. 1. A family of Newton trajectories on Müller-Brown (MB) PES [17].

desingularized Newton equation, see Refs. [6,7,13,15], which is a special form of the well known NewtonRaphson method.

\section{Proposition 1. [15] It holds}

1. $\mathbf{x} \in \operatorname{Ess}(\mathscr{K})$ is equivalent that for all $r \in S^{n-1}$ it is $\mathbf{x} \in \mathscr{T}_{r}(\mathscr{K})$, which is also equivalent that for $r, s \in$ $S^{n-1}$, and $r \neq s$ it is $\mathbf{x} \in \mathscr{T}_{r}$ and $\mathbf{x} \in \mathscr{T}_{s}$,

2. from $\mathbf{x} \in \mathscr{K} \backslash \operatorname{Ess}(\mathscr{K})$ it follows that $\mathbf{x} \in \mathscr{T}_{G(\mathbf{x}) \|}\|G(\mathbf{x})\|$

3. from $\operatorname{Ess}(\mathscr{K}) \neq \emptyset$ it follows that $\mathscr{T}_{r}(\mathscr{K}) \neq \emptyset$, for all $r \in$ $S^{n-1}$.

\subsection{Branches and components of Newton trajectories}

NTs are smooth, parameterizable curves in nonstationary points, where the rank of the matrix $\partial_{x} \mathscr{R}(\mathbf{x}, r)=\mathscr{P}_{r} H(\mathbf{x})$ is $n-1$ (i.e. maximal) [18]. It follows from the implicit function theorem. The condition is fulfilled if the rank of the augmented Hessian $[H, G]$ is maximal.

Definition 8. The set $\operatorname{Ext}(\mathscr{K})$ of Eq. (3) is the set of extraneous singularities. All points in $\mathscr{K}$, which are neither in $\operatorname{Ess}(\mathscr{K})$ nor in $\operatorname{Ext}(\mathscr{K})$, are named regular.

The characterization of a nondegenerate point uses properties of the PES; but the characterization of a regular point uses properties of Newton trajectories. An inflection point, where the gradient points in direction of the zero eigenvector, is degenerate, however, in the general case, it is 


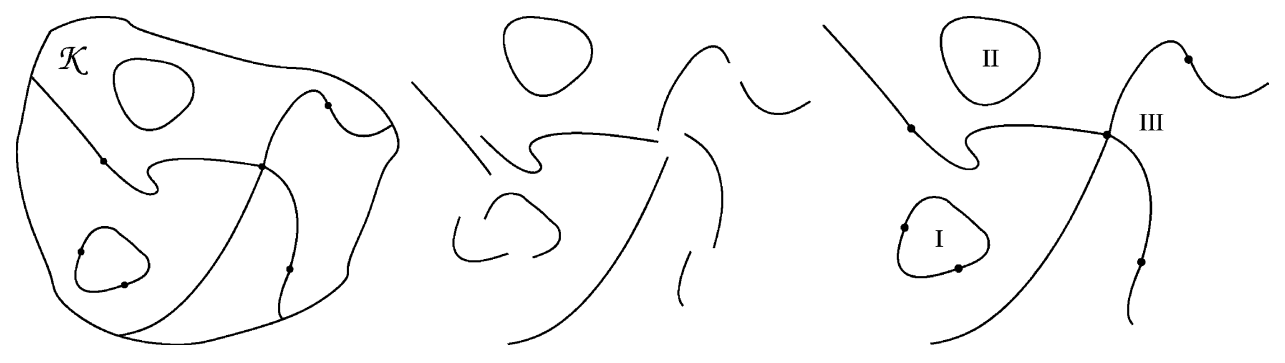

Fig. 2. A Newton trajectory, its branches and components.

a regular point of the corresponding NT. If one takes off the singular points of an NT, one will get a set of smooth, parameterizable pieces of curves, see Fig. 2. Any such piece of a curve will be named a branch. The end points of open branches are the points of the boundary of $\mathscr{K}$ or singular points. The NT of Fig. 2 is partitioned into three components: I and II are compact sets in the interior of $\mathscr{K}$, but III is open in $\mathscr{K}$.

Component I contains two StPs, but component II does not contain any StP.

Preposition 2. [19] Every compact component of a Newton trajectory, being disjunct to the boundary of $\mathscr{K}$, contains an even number or no stationary points.

Caution. The word 'compact' in the hypothesis is crucial! Fig. 3 shows the four NTs which cross the VRI points of the MB potential; the VRIs themselves are given in Table 1. The figure top right contains a compact component
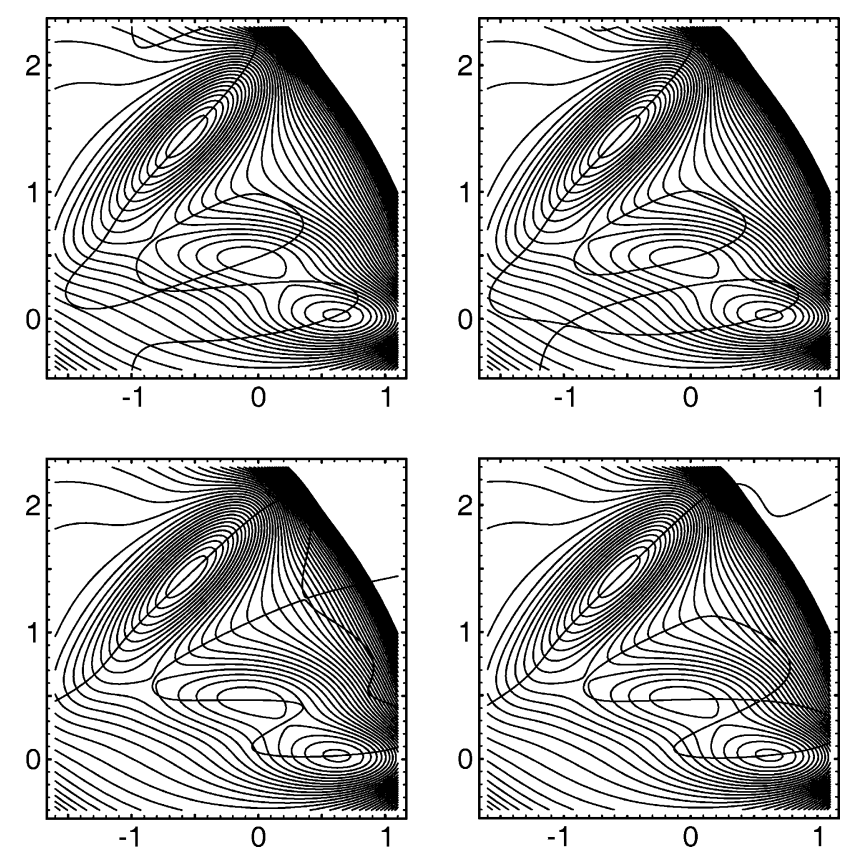

Fig. 3. Singular NTs of the MB PES [17] which cross the VRIs. Corresponding search directions are $66.805^{\circ}, 61.96^{\circ}, 30.39^{\circ}, 37.67^{\circ}$ (from top left to bottom right). of an NT with two StPs, and the figure bottom right has a compact component of an NT which crosses four StPs.

\subsection{Branin's method}

The reduced gradient approach shows an analogy to the mathematical theory of Branin [20], the global Newton method [15]. It utilizes the adjoint matrix $A$ of the Hessian matrix $H$. This is defined as $\left((-1)^{i+j} m_{i j}\right)^{T}$ where $m_{i j}$ is the minor of $H$ obtained by deletion of the $i$ th row and the $j$ th column from $H$, and taking the determinant. The adjoint matrix satisfies the relation

$H A=\operatorname{det}(H) I$,

where $\operatorname{det}(H)$ is the determinant of $H$, and $I$ is the unit matrix. The adjoint matrix $A$ is used to define an autonomous system of differential equations for the curve $\mathbf{x}(s)$, where $s$ is a curve parameter

$\frac{\mathrm{d} \mathbf{x}(s)}{\mathrm{d} s}=A(\mathbf{x}(s)) G(\mathbf{x}(s))$.

Proposition 3. [6] Solutions of the Branin Eq. (12) are branches of Newton trajectories.

\section{Extraneous singularities}

In this section we add a more detailed treatment to the Definition 4 of VRI points, see Eq.(3). We use results of Jongen et al. [15] and Diener [19].

On a 2D PES the VRI points are single, isolated points. Thus, they form a 'zero-dimensional manifold'. For an $n$-dimensional PES the VRI points can be a manifold of a dimension up to $n-2$. Such manifolds are found, in the special case of symmetric VRI points, for the PES of

Table 1

VRI points on MB-PES, and search direction of NTs

\begin{tabular}{llrr}
\hline Point & Direction & \multicolumn{1}{l}{} & \multicolumn{1}{l}{$y$} \\
\hline $\mathrm{VRI}_{1}$ & $66.805^{\circ}$ & -0.75002 & 0.22586 \\
$\mathrm{VRI}_{2}$ & $61.96^{\circ}$ & -0.98072 & -0.04753 \\
$\mathrm{VRI}_{3}$ & $30.39^{\circ}$ & 0.37250 & 1.26315 \\
$\mathrm{VRI}_{4}$ & $37.67^{\circ}$ & 0.54859 & 0.45930 \\
\hline
\end{tabular}




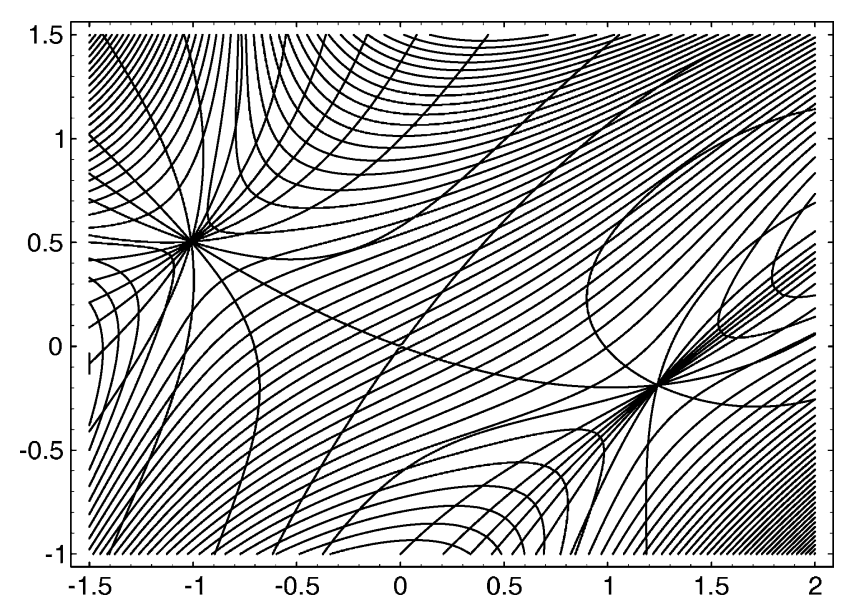

Fig. 4. Index theorem. The surface shows two adjacent SPs of index one. There is no regular branch of an NT connecting the SPs. Between the SPs a VRI point has to exist. The singular NT is shown which leads to the VRI point and branches there.

water by Hirsch et al. [21], for formaldehyde by Quapp and Melnikov [22], and for $\mathrm{C}_{2} \mathrm{H}_{5}^{+}$by Quapp and Heidrich [23].

Note. It is a fundamental property that the VRIs are not isolated points in higher dimensions. In theoretical chemistry it is practice to report single VRI points, cf. Ref. [13] and a legion of references therein. However, in real molecules, these points are part of the high-dimensional manifold of VRI points. The existence of a full manifold of VRIs includes the question for the meaning of special points for an RP branching. Thus, again, it seems that a single curve as RP definition becomes questionable. Below, we treat a whole family of NTs for a reaction channel. Thus, we study the topology of a PES. The discussion again shows that 2D pictures are often fine examples, but not enough to understand the high-dimensional PESs of real molecules.

A VRI point in two dimensions is characterized by a 1-dimensional kernel of the reduced Hessian $\mathscr{P}_{r} H(\mathbf{x})$. Thus, there are two tangents to the NT at $\mathbf{x}$. For a general PES it is possible that the rank of $\mathscr{P}_{r} H(\mathbf{x})$ further reduces. Such a VRI point can be the carrier of more than two tangents. The amount of the rank deficit of $\mathscr{P}_{r} H(\mathbf{x})$ produces a $k$-fold singularity, or a $k$-fold VRI point. The maximal dimension of the set of VRI points of a special multiplicity $k$ decreases with the multiplicity. On the PES of formaldehyde there are already at least two-fold VRI points [22].

Proposition 4. (Index theorem [19]) Let $\mathbf{x}_{1}$ and $\mathbf{x}_{2}$ be stationary points connected by a regular branch of a Newton trajectory. Then it holds

$\operatorname{ind}_{2}\left(\mathbf{x}_{1}\right) \neq \operatorname{ind}_{2}\left(\mathbf{x}_{2}\right)$.

A regular branch of a Newton trajectory connects an StP of an odd index and an StP of an even index. The index theorem has direct practical use, see Fig. 4. If one numerically follows the branch of an NT, and one connects StPs of a 'false' index by the procedure then one has a tool to detect the error. An example of such an error can be found for the molecular fragmentation of $C_{4} H_{10}$ in Ref. [24] where in Fig. 6 the pathways between different StPs are given by NTs. Some connections are drawn between false index numbers.

At least one change of the index of a regular NT is given by an inflection point of the energy profile. Every continuous connection of two StPs has at least one such inflection point. The extraneous singularity breaks the index along an NT by a further odd number (by 1), see Fig. 5: a second change of the index happens at the VRI point. We find the index transformations $0-2$ and $1-1^{\prime}$. Around the VRI point there are regular branches of neighboring NTs which connect $0-1^{\prime}$ and $1-2$, or $0-1$ and $1^{\prime}-2$, correspondingly. These trajectories are to grasp by a separation of the center case at the VRI point.

\subsection{Phase portraits}

The word creation 'valley-ridge inflection' point is coined by the $2 \mathrm{D}$ imagination of a valley branching. Such a singularity has a phase portrait of saddle type, see Figs. 6 and 7. The imagination in the $n$-dimensional case is
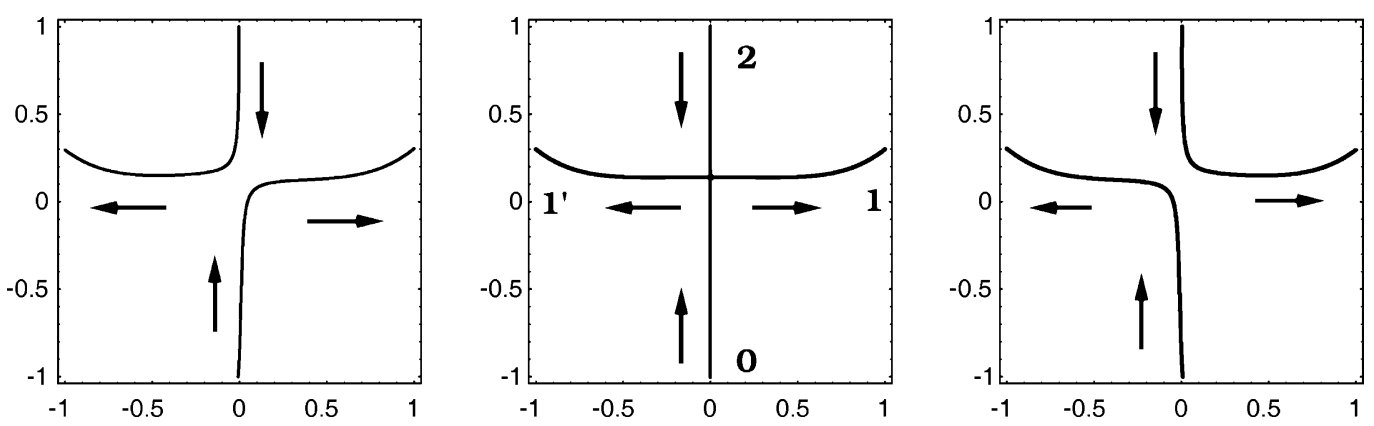

Fig. 5. Index theorem. Typical scheme for the relation of StPs with different index surrounding the VRI point of an NT (center). Side parts: neighboring regular NTs. 


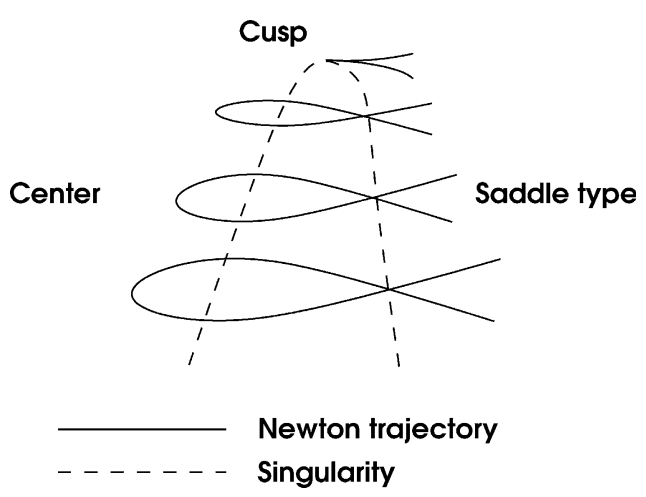

Fig. 6. Phase portraits of Newton trajectories.

difficult. For 3D there are three different types: center type and saddle type, and for the transition between both the cusp type.

\subsection{Example: VRI points along an NT on the PES of formaldehyde}

The PES of formaldehyde is calculated with the restricted Hartree-Fock method (RHF) and STO-3G basis set. There are many StPs up to SPs of index 3 [5,25]. The example NT starts with the global minimum and follows the search direction of the symmetric bending. The NT leads to an SP of index 3, see Fig. (8) and Table 2 [26].

Along the NT there are two VRI points.

At step 11 of the predictor for the NT of Fig. 8 the eigenvalue crosses zero which belongs to the change of the dihedral angle $\angle \mathrm{H}_{\alpha} \mathrm{COH}_{\beta}$. The branching breaks the $C_{2 v}$ symmetry to $C_{S}$ symmetry. Along the branches the dihedral angle changes under mirror symmetry: $r_{\mathrm{H}_{\alpha} \mathrm{C}}=r_{\mathrm{H}_{\beta} \mathrm{C}}$ and $\angle$ $\mathrm{H}_{\alpha} \mathrm{CO}=\angle \mathrm{H}_{\beta} \mathrm{CO}$. Fig. 9 is a section of the PES of $\mathrm{H}_{2} \mathrm{CO}$ with the VRI point. The $y=0$ line corresponds to a small piece of the NT of Fig. 8. It is fixed at $1.1 \AA r_{\mathrm{CH}_{\alpha}}=r_{\mathrm{CH}_{\beta}}$, and the dihedral angle varies between $90^{\circ}$ and $270^{\circ}$, and it is used on the $y$ axis in a scaling where the zero corresponds to the planar symmetry at $180^{\circ}$. We use angles $\alpha_{\mathrm{H}_{\alpha} \mathrm{CO}}=\alpha_{\mathrm{H}_{\beta} \mathrm{CO}}$ and $r_{\mathrm{CO}}$ at: $\alpha_{\mathrm{HCO}}=125-\mathrm{i} \cdot 3$ or $r_{\mathrm{CO}}=1.2+\mathrm{i} \cdot 0.03$, correspondingly, for parameter $i$ between 0 and 20 being the $x$ axis. Thus, the angles $\alpha_{\text {HCO }}$ vary between $125^{\circ}$ and $65^{\circ}$, while at the same time the CO-distance varies in 1.2 and $1.8 \AA$.
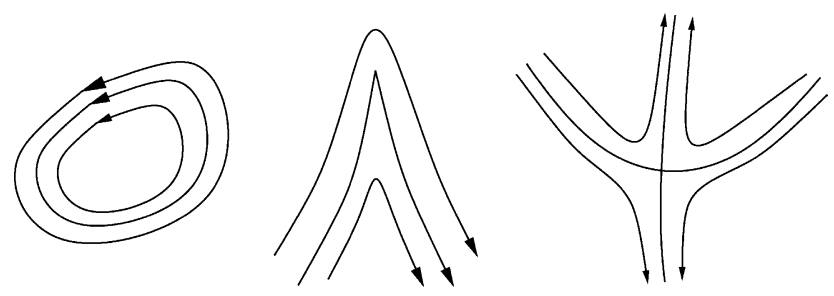

Fig. 7. Phase portraits of NTs around extraneous singularities. From left to right: center type, cusp type and saddle type.

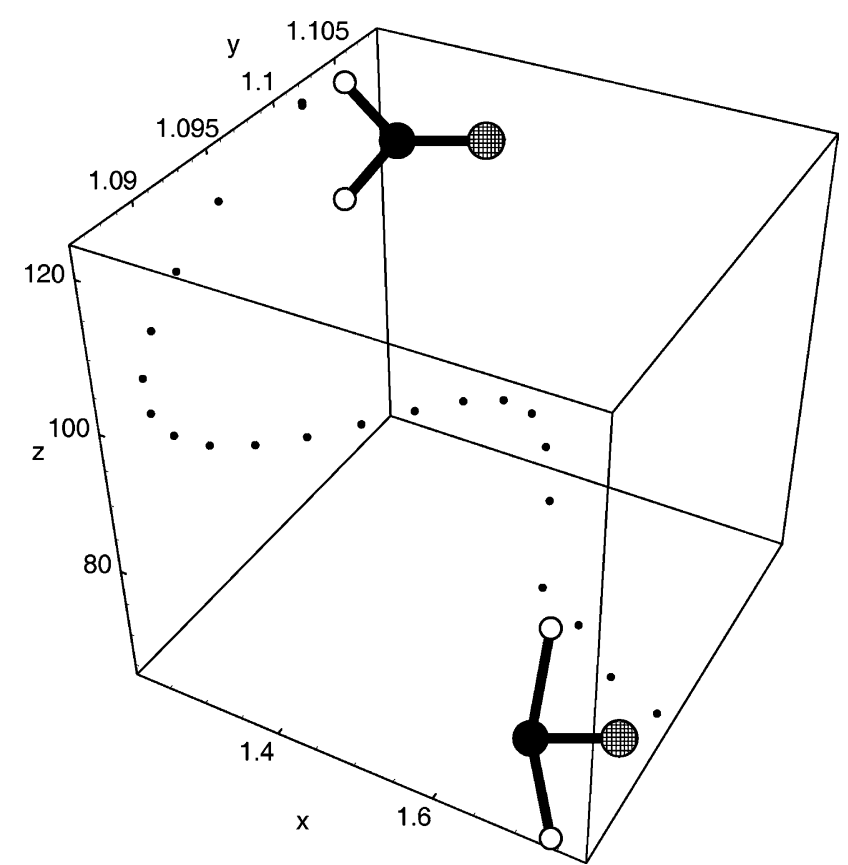

Fig. 8. Newton trajectory on PES of $\mathrm{H}_{2} \mathrm{CO}$ (RHF STO-3G). The path is given in reduced configuration space with $\mathrm{x}=\mathrm{r}_{C O}, \mathrm{y}=\mathrm{r}_{H C}, \mathrm{z}=\angle \mathrm{HCO}$ and with $C_{2 v}$ symmetry. The NT goes from global minimum (top) to an SP of index 3 (bottom).

At step 16 along the calculated NT of Fig. 8 the eigenvalue crosses zero which belongs to the change of the antisymmetric angle. It is shown in Fig. 10. The branching of this VRI point breaks the $C_{2 v}$ symmetry to $C_{2}$ symmetry. On the branches the dihedral angle is fixed at $180^{\circ}$, but the angles $\angle \mathrm{H}_{\alpha} \mathrm{CO}$ and $\angle \mathrm{H}_{\beta} \mathrm{CO}$ change in opposite directions. Fig. 10 shows the region around the VRI point. The distance $r_{\mathrm{CH}}$ is fixed at $1.1 \AA$, and the $x$ axis is the symmetric change of the angle $\alpha_{\mathrm{HCO}}$ and the distance $r_{\mathrm{CO}}$ like in Fig. 9. The dihedral angle is fixed at $180^{\circ}$. $y$ axis is the symmetry change of angle $\alpha_{\mathrm{HCO}}$ in degree. The $y=0$ line corresponds to a small piece of the NT of Fig. 8 .

Figs. 9 and 10 are 2D sections of the 6-dimensional PES of formaldehyde, and that is why they allow only a restricted overview of the full PES. Especially, the trend of the changes of $\alpha_{\mathrm{HCO}}$ and $r_{\mathrm{CO}}$ is linearized. It is not fully the region which is covered by the curvilinear NT of Fig. 8. But the pictures give a good insight into the 'instability' of the $C_{2 v}$ symmetry in this region of the PES. Note that along the curve in Fig. 8 the energy strongly monotonously increases from the global minimum left-above to the SP of index 3 'right-below'.

Table 2

Two stationary points on PES of $\mathrm{H}_{2} \mathrm{CO}$ connected by an NT [5]

\begin{tabular}{llllll}
\hline Index & Energy (a.u.) & Symmetry & $r_{\mathrm{CO}}(\AA)$ & $\mathrm{r}_{\mathrm{CH}}(\AA)$ & $\alpha_{\mathrm{HCO}}($ deg.) \\
\hline 0 & -112.3544 & $C_{2 v}$ & 1.217 & 1.101 & 122.74 \\
3 & -112.0122 & $C_{2 v}$ & 1.770 & 1.095 & 65.44 \\
\hline
\end{tabular}



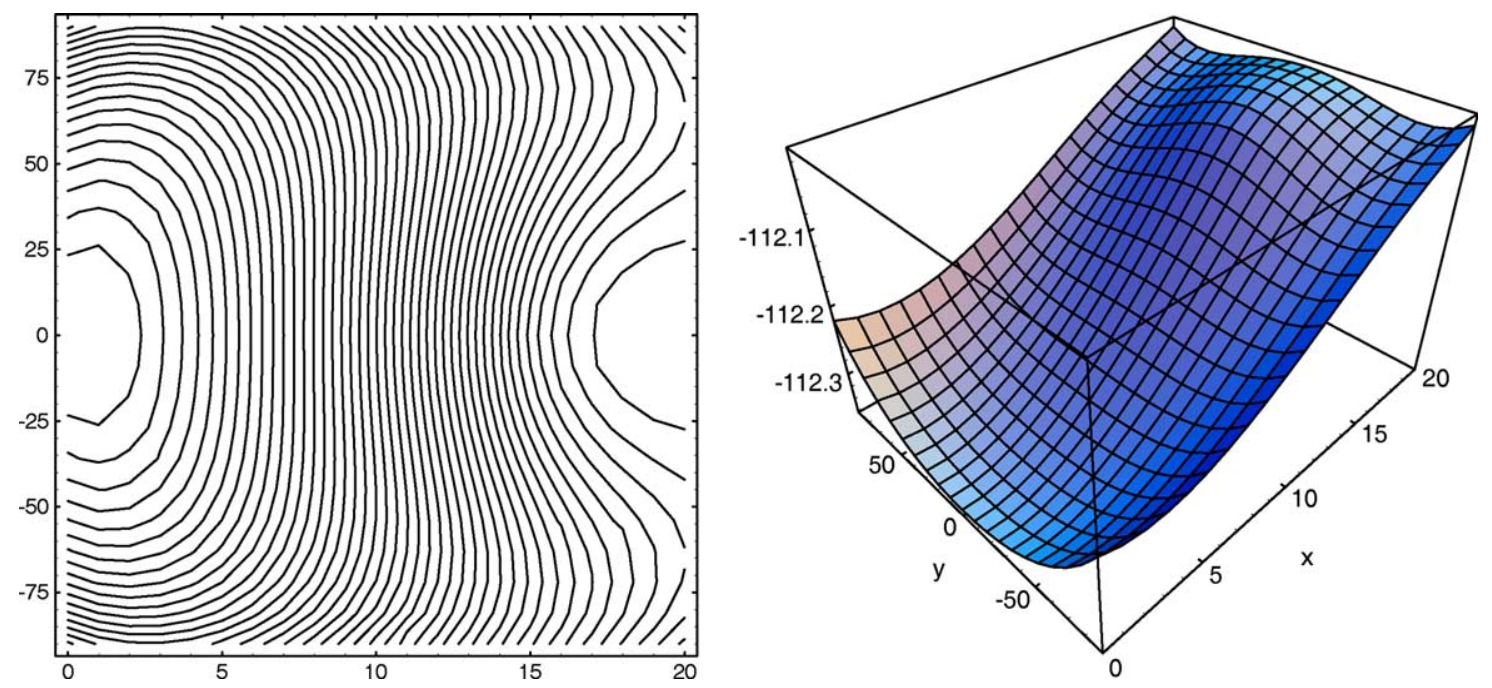

Fig. 9. PES section of $\mathrm{H}_{2} \mathrm{CO}$ with VRI. $y$ axis is dihedral angle, $x$ axis is change of $r_{\mathrm{CO}}$ and the symmetric $\alpha_{\mathrm{HCO}}$, see text. At VRI point the valley branches uphill and breaks the $C_{2 v}$ symmetry to $C_{S}$ symmetry.

\section{Newton channels}

With Proposition 1 every StP is on all NTs.

Definition 9. We will say two stationary points are adjacent if they are connected by a regular branch of an NT.

Now, we look for all branches which start at a fixed StP, $A$. Which part of that family connects $A$ with other StPs? To any branch beginning at $A$ belongs a direction $r \in S^{n-1}$. Thus, since we fix $A$, we can turn the point of view to $S^{n-1}$, if we identify the directions $r$ with their corresponding branches by the help of the trajectory map $\underline{r}=G /\|G\|$ of Definition 6. We put $A$ for the initial point of all branches, and identify the search directions of all these NTs with all points of $S^{n-1}$. Every branch has three possible kinds of final point: (i) an StP with an index difference of 1 to $A$, or (ii) a VRI point, or (iii) a point of the border of the configuration space. The VRI points can form pieces of a manifold of a dimension up to $(n-2)$. Branches which meet the VRI points are singular branches. They form a 1-codimensional submanifold on $S^{n-1}$. It divides the into a disjunct system of open sets. All points of one of the sets correspond to NTs which lead to the same StP, say $B$, or to the border of $\mathscr{K}$. The border of the sets of $S^{n-1}$ is formed only from singular directions.

Definition 10. Regular branches of different Newton trajectories will be named equivalent if they can be
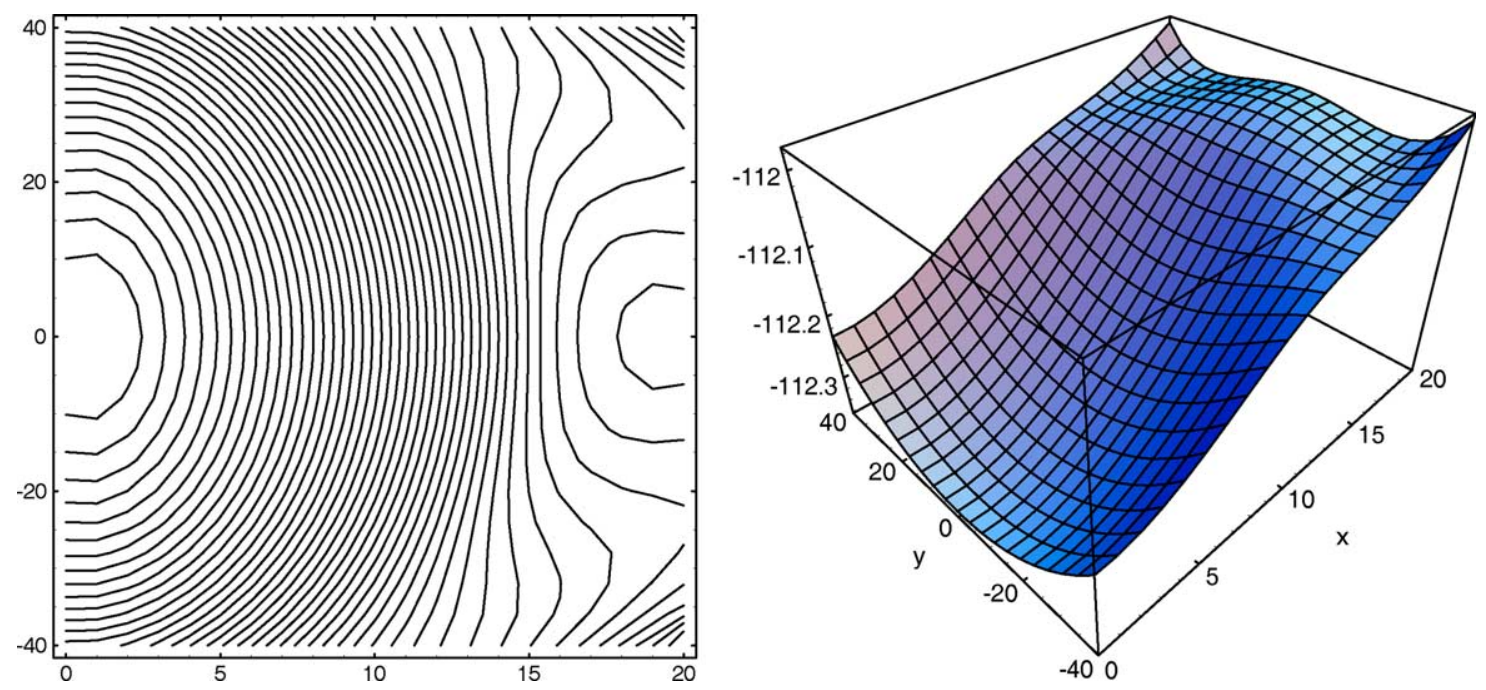

Fig. 10. PES section of $\mathrm{H}_{2} \mathrm{CO}$ with VRI. $y$ axis is deviation of the symmetry of the angles $\alpha_{\mathrm{HCO}}, x$ axis is parameter with $r_{\mathrm{CO}}$ and $\alpha_{\mathrm{HCO}}$, see text. At VRI point the valley branches and breaks the $C_{2 v}$ symmetry to $C_{2}$ symmetry. 
transformed from one to the other without meeting a singular branch, where the transformation is a continuous variation of the search direction. The corresponding equivalence class is the Newton channel.

Caution. The word 'regular' in the definition is crucial! The Newton channels starting in one StP are divided by walls formed by singular branches. The walls are 'thin' because they have a lower dimension. If the volume of $S^{n-1}$ is 1 then the sum of the volumina of all sets of regular trajectories is 1 , however, the volume of the partitions between the sets is zero. The walls are a separatrix and there is a disconnection of the sets formed by the regular NTs. An NT starting at a point that is strictly on a wall will never leave the border (by definition). The walls lie in the closure of their corresponding channels. The StP, to which a channel leads, has to belong to the wall of the channel as well, thus, to the component of connection of the singular NTs forming the wall. Singular NTs usually lead to at least three StPs because they bifurcate at a VRI point.

We interpret the Newton channels as approximations of reaction channels. Fig. 11 is a model of the region around reactant $A$ for reactions $\mathrm{A} \rightarrow \mathrm{P}_{1}$ over the transition structure $\mathrm{B}$ and $\mathrm{A} \rightarrow \mathrm{P}_{2}$ over the transition structure $\mathrm{C}$, taking place from the same reactant $A$ to the product systems $P_{1}$ and $P_{2}$, and so on. To every reaction belongs one reaction channel, and the channels are divided by thin walls. Pathways going on in the walls meet a bifurcation from which branches lead aside to the two transition structures. Thus, the singular NTs of the wall between B and C belong to both SPs. A pathway of the wall can be calculated: if a regular NT to direction $r_{0}$ leads from minimum A to SP B, and if a further regular NT to direction $r_{1}$ leads from minimum A to SP C, then there exist a $\lambda \in(0,1)$, such that $r_{\lambda}=R_{\lambda} /\left\|R_{\lambda}\right\|$ belongs to a singular NT, and $R_{\lambda}=\lambda r_{0}+(1-\lambda) r_{1}$ is a convex combination of $r_{0}$ and $r_{1}$, see Fig. 11. One may use an interval inclusion to find the singular direction $r_{\lambda}$. However, numerically following a singular NT is difficult near its VRI

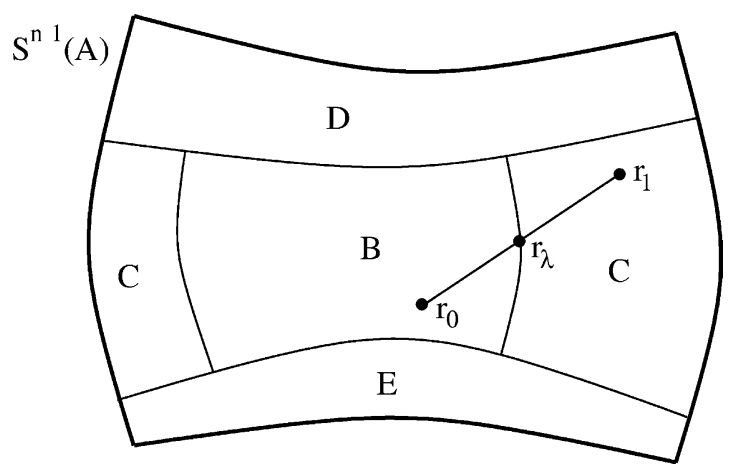

Fig. 11. Scheme of $S^{2}$ for the Newton channels on $S^{n-1}$ starting at the StP A. The representation is opened like a map of the world in Mercator projection [27]. Points B to E depict the sets of different search directions belonging to the Newton channel, respectively. Every Newton channel leads to one $\mathrm{StP}(\mathrm{B}, \mathrm{C}, \mathrm{D}$ or $\mathrm{E})$. If $\mathrm{A}$ is minimum then the points $\mathrm{B}$ to $\mathrm{E}$ will be SPs of index one. point, because the numerical methods break down [13] (see also Fig. 17 below).

To every point of the PES belongs an NT. Thus, it is possible to divide the PES into Newton channels, totally. All channels are partitioned by thin walls of singular branches of NTs. Theoretically, there can be channels without StPs. Theoretically, the system of channels needs not be simply connected. However, this does not seem to be of interest for chemical applications. All about 50 StPs of Ref. [5] for formaldehyde are adjacent, in any kind vice versa, in a net of points. They are calculated by following all the branches beginning from every StP into the 12 coordinate directions. It emerges that we can connect the StPs of a PES by Newton channels in form of a graph [28].

\section{Conclusion}

A PES can be represented as a Newton graph: vertices are the StPs, edges are the Newton channels. With the index theorem it follows that a Newton graph cannot contain circles with an odd number of edges. Further, Newton graphs do not have loops (edges connecting one vertex with itself).

To any Newton channel belongs the volume of the induced tangents [7]. We can assign to every edge a weight by the volume of the Newton channel. It is the measure of the set of directions of $S^{n-1}$, the NTs of which have branches in this Newton channel. The theoretical case of closed channels is not of interest for reaction theories.

In this kind we can assign many NTs, or few NTs to a region of the PES! It is not trivial because to every point of the PES belongs one NT. The 'density' of NTs differs from region to region. The trajectory map $\underline{r}$ of Definition 6 is a diffeomorphism of an open subset of an equipotential surface $\mathscr{E}_{\bar{x}}$, around a point $\overline{\mathbf{x}}$, into $S^{n-1}$, if the pseudoconvexity index

$$
\xi: \mathscr{K} \backslash \operatorname{Ess}(\mathscr{K}) \rightarrow \mathbb{R}, \quad \xi(\mathbf{x}):=\frac{G(\mathbf{x})^{T} A(\mathbf{x}) G(\mathbf{x})}{G(\mathbf{x})^{T} G(\mathbf{x})}
$$

is not zero on this subset [7,29]. If the volume of the image of $\mathscr{E}_{\bar{x}}$ is small then less NTs meet $\mathscr{E}_{\bar{x}}$. If the volume of the image of $\mathscr{E}_{\bar{x}}$ is large then more NTs meet $\mathscr{E}_{\bar{x}}$. All this description may seem rather baroque, given that most points are not on walls.

(However note, the imagination of volume in higher dimensions is treacherous. Most of the volume of a higherdimensional hypersphere is associated with the surface [30]. Thus, most of the configuration space in a large chemical system must lie near the equipotential surface of the corresponding potential energy, cf. [31]. Or, if we think in terms of the singular 'walls', most of the 'space of all NTs' in $S^{n-1}$ will lie near the walls which partition the regular sets. This happens at least on the pieces where the singular 
NT goes parallel to a valley floor. However, the situation may be something more complicated than in the case of descent lines, cf. [32], because at a VRI point, where the singular NT bifurcates, the curvature of the equipotential level of interest is exact zero. There the 'density of the NTs' in relation to $S^{n-1}$ is very small.)

It may be a conclusion that we can define an RP as the region of the PES where the density of NTs is high and the PES forms a valley. The definition will be useful at least in the cases where the SP is on the top of the valley, and where the RP does not bifurcate, cf. [33]. In these cases the imagination of a reaction channel is well adapted to reality.

\subsection{Example Müller-Brown potential}

It is possible to fully study the 2D PES [17] with NTs. One may numerically follow an NT along its branches. We begin at the global minimum $M_{1}$ in all directions $(r \in$ $\left[-180^{\circ}, 180^{\circ}\right)$ or $\left.r \in\left[0^{\circ}, 360^{\circ}\right)\right)$, correspondingly. Figs. 1 and 3 give some examples. In Fig. 1 the distance of the search directions from one trajectory to the next is always equal. It can be observed that the trajectories concentrate in valleys, and on ridges. It can be seen that the more NTs lead through a region the more the equipotential surfaces are curved. This effect will grow dramatically in higher dimensions [30,32]. Exactly this effect is also used in the TASC method [34], the tangent search concept of a gradient extremal (GE): the GE following is replaced by an approximate following of the valley family of NTs which go parallel along the valley floor.

Now we treat the Newton trajectories to all search directions in the interval $\left[0,180^{\circ}\right), \mathscr{K}=(-1.6,1.1) \times$ $(-0.4,2.3)$. Four NTs meet singular points (see Figs. 3

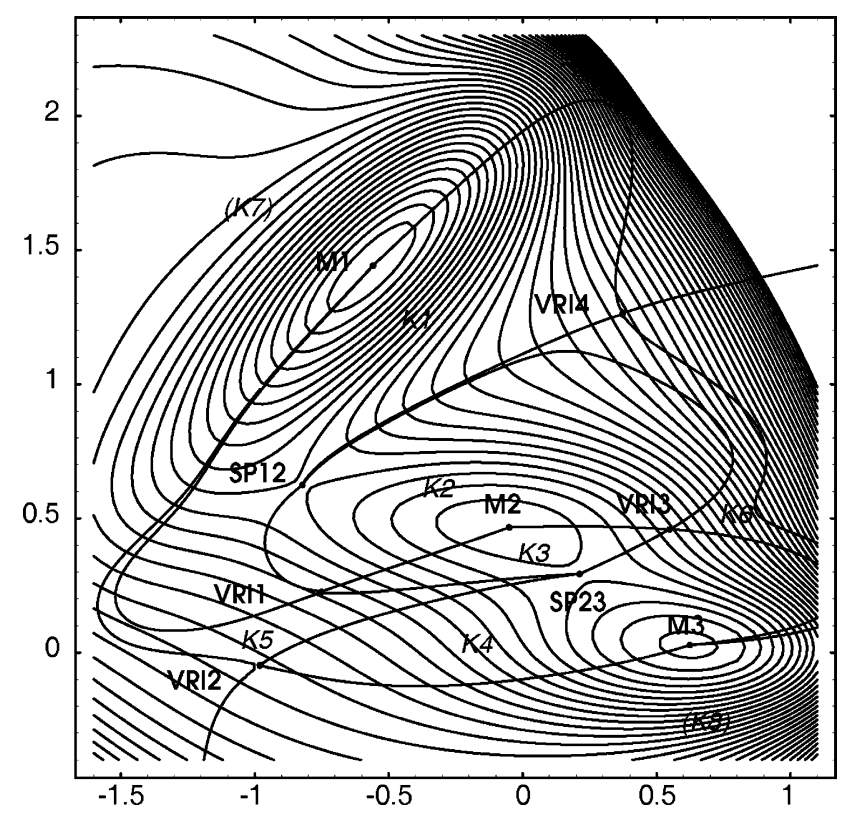

Fig. 12. Singular branches of Newton trajectories on Müller-Brown potential dividing the configuration space in channels.
Table 3

Intervals, size and ratio of $360^{\circ}$ of classes of regular NTs, $\tau_{i}$, on MüllerBrown potential in Fig. 13. (Cf. also Fig. 3 and Ref. [14])

\begin{tabular}{llll}
\hline$\tau_{i}$ & Interval $\left(r_{\min }, r_{\max }\right)$ & $\Delta r$ & $\Delta r / 360^{\circ}$ \\
\hline 1 & $\left(66.805^{\circ}, 30.39^{\circ}\right)$ & $143.6^{\circ}$ & 0.399 \\
2 & $\left(30.39^{\circ}, 37.67^{\circ}\right)$ & $7.3^{\circ}$ & 0.020 \\
3 & $\left(37.67^{\circ}, 61.96^{\circ}\right)$ & $24.3^{\circ}$ & 0.068 \\
4 & $\left(61.96^{\circ}, 66.805^{\circ}\right)$ & $4.8^{\circ}$ & 0.013 \\
\hline
\end{tabular}

and 12 and Table 1). The other NTs belong to four sets of the topological equivalence classes of regular NTs. The singular branches of MB potential are the walls (here lines) of the Newton channels. The classes of regular branches, which connect two stationary points, respectively, are shown summarily in Fig. 12. Newton channels are depicted by $\mathrm{K} 1$ to $\mathrm{K} 8$, where especially the channels $\mathrm{K} 7$ and $\mathrm{K} 8$ lead from a minimum to the border of $\mathscr{K}$. The largeness of a Newton channel is the size of the class of regular NTs, see Table 3. The sum of all channels beginning in one StP is 1 $\left(360^{\circ}\right.$, in the $2 \mathrm{D}$ case). The Newton channels of MüllerBrown potential are given in Table 4 .

In Fig. 13 four representatives are shown, respectively. Class 1 is best suited for the search of StPs. It connects all StPs with one line, one connected component. Class 1 has a measure of $143.6^{\circ}$ from $180^{\circ}$, see Table 3. Fig. 14 depicts the qualitative development of NTs depending on the search direction. A representative of every regular set is shown, and these are divided by the special, singular NTs, see also Table 3. All VRI points of $\mathscr{K}$ are included.

Figs. 12 and 15 show, that the Müller-Brown potential is separated into an internal and an outer region of NTs, correspondingly. The outer region itself is again separated into two parts. It is covered with regular trajectories only from the minima M1 and M3. Starting at minimum M2, two Newton channels lead only to the SPs S12 and S23. Starting

Table 4

Newton channels on MB potential. Region and size are seen from the corresponding minimum. Classes are from Table 3

\begin{tabular}{|c|c|c|c|c|}
\hline & Start & Classes & Region & Size \\
\hline K1 & M1 & $\tau 1$ & $\left(-113.195^{\circ}, 30.39^{\circ}\right)$ & 0.399 \\
\hline K5 & M1 & $\tau_{4}$ & $\left(-118.04^{\circ},-113.195^{\circ}\right)$ & 0.013 \\
\hline K7 & M1 & $\tau 1+2 \tau_{2}+2 \tau_{3}+\tau_{4}$ & $\left(30.39^{\circ}, 241.96^{\circ}\right)$ & 0.588 \\
\hline $\mathrm{K} 2$ & M2 & $\tau_{1}+\tau_{2}+2 \tau_{3}+2 \tau_{4}$ & $\left(37.67^{\circ}, 241.96^{\circ}\right)$ & 0.567 \\
\hline K3 & M2 & $\tau_{1}+\tau_{2}$ & $\left(-118.04^{\circ}, 37.67^{\circ}\right)$ & 0.434 \\
\hline K4 & M3 & $\tau_{1}+\tau_{2}+2 \tau_{3}+\tau_{4}$ & $\left(37.67^{\circ}, 246.805^{\circ}\right)$ & 0.581 \\
\hline K6 & M3 & $\tau_{2}$ & $\left(30.39^{\circ}, 37.67^{\circ}\right)$ & 0.020 \\
\hline K8 & M3 & $\tau_{1}+\tau_{4}$ & $\left(-113.195^{\circ}, 30.39^{\circ}\right)$ & 0.399 \\
\hline
\end{tabular}



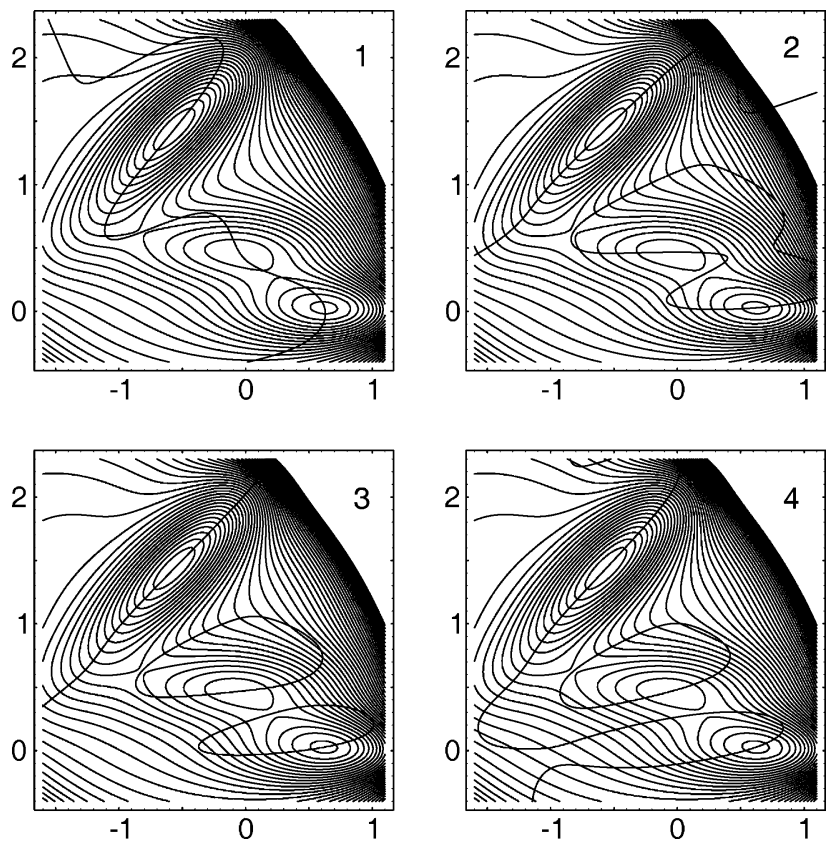

Fig. 13. Representatives of the 4 classes of regular NTs, see Table 3.

at the minima M1 and M3 a main channel leads to direction of M2 (K1 with $39.9 \%$ of all branches, or K4 with $56.8 \%$ of all branches). Side channels (K5 with $1.3 \%$, or K6 with $2 \%$ ) 'jump over' the central minimum M2.
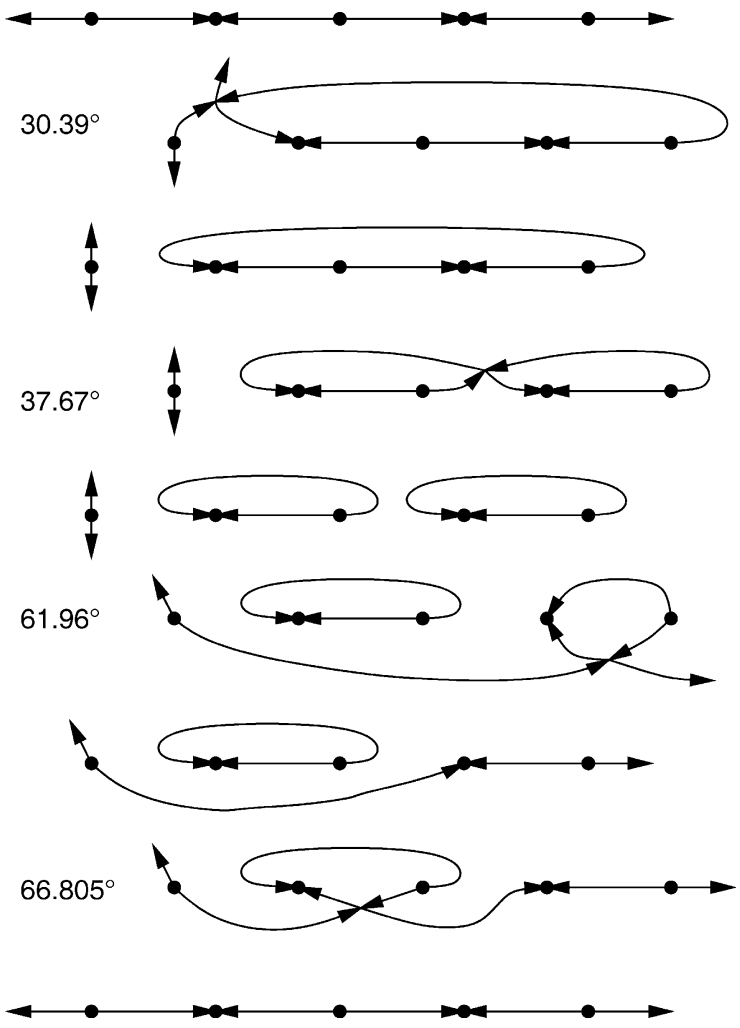

Fig. 14. Scheme of consecutive regular and singular NTs on Müller-Brown potential. Bullets are stationary points.

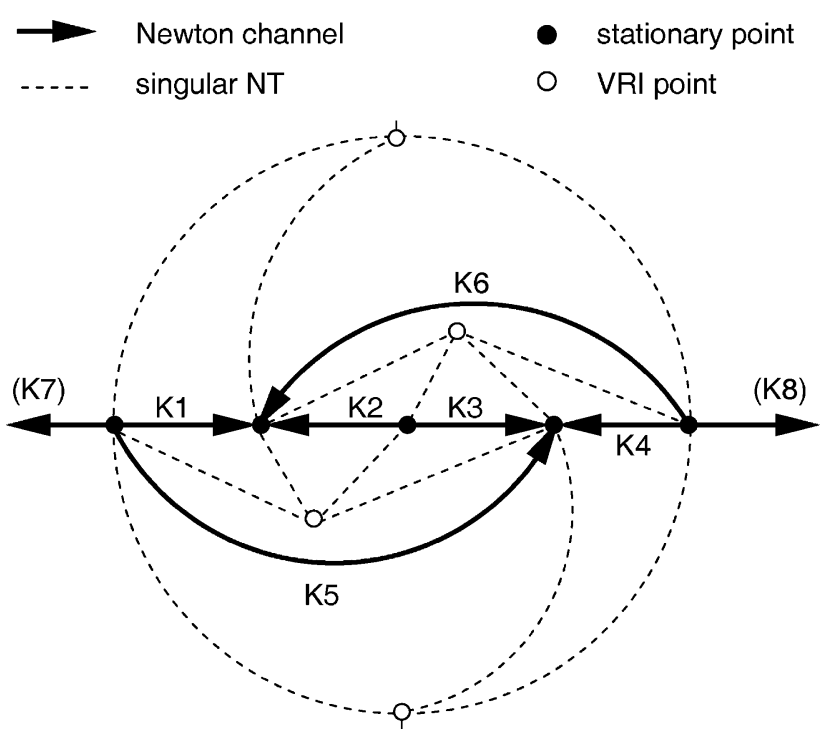

Fig. 15. Graph of Newton channels (arcs are pointer to SPs), and singular Newton trajectories on Müller-Brown potential. Vertices are minima and SPs.

\subsection{Example $\mathrm{H}_{2} \mathrm{O}$}

The example is the MP2/6-31G** PES of water, see Fig. 16 and also Fig. 8b of Ref. [21]. It is depicted by A the global minimum of $\mathrm{H}_{2} \mathrm{O}$, by $\mathrm{B}$ the dissociation to $\mathrm{O}+\mathrm{H}_{2}$, by $\mathrm{C}$ the dissociation to $\mathrm{O}+\mathrm{H}+\mathrm{H}$, by $\mathrm{D}$ the linear saddle point $\mathrm{H}-\mathrm{O}-\mathrm{H}$ and by $\mathrm{E}$ or $\mathrm{E}^{\prime}$ the dissociations to $\mathrm{OH}_{\alpha}+\mathrm{H}_{\beta}$, or $\mathrm{OH}_{\beta}+\mathrm{H}_{\alpha}$. Newton channels belong to regular trajectories $\mathrm{A} \rightarrow \mathrm{D}, \mathrm{A} \rightarrow \mathrm{E}$, and $\mathrm{A} \rightarrow \mathrm{E}^{\prime}$, which have to be partitioned by singular directions on their representation on $S^{2}\left(\mathrm{H}_{2} \mathrm{O}\right)$ (i.e. $S^{2}(\mathrm{~A})$ ). The singular directions which divide the channels to $\mathrm{E}$ and $\mathrm{E}^{\prime}$ are the symmetric search directions with $r\left(\mathrm{OH}_{\alpha}\right)=r\left(\mathrm{OH}_{\beta}\right)$. Assigned to these directions is the symmetric manifold of VRI points which is reported in Ref. [21], and there it is depicted by $\mathrm{VRI}_{1}$ in Fig. 8b. But there has to be a wall between the channels $\mathrm{D}$ and $\mathrm{E}$, or $\mathrm{D}$ and $\mathrm{E}^{\prime}$ also, see Fig. 16 right.

In this wall a further 1-dimensional VRI manifold lies which crosses the symmetry plane at $r\left(\mathrm{OH}_{\alpha}\right)=$ $r\left(\mathrm{OH}_{\beta}\right) \approx 1.2 \AA$ and $\alpha(\mathrm{HOH}) \approx 140^{\circ}$. We try to find this wall. We use a family of NTs from minimum A, see Fig. 17. One part of the NTs goes to the linear SP, D, it belongs to Newton channel $\mathrm{A} \rightarrow \mathrm{D}$. The other part of the NTs leads to dissociation $\mathrm{OH}+\mathrm{H}$, it belongs to channel $\mathrm{A} \rightarrow \mathrm{E}\left(\right.$ or $\left.\mathrm{E}^{\prime}\right)$. One calculated line with marked path points leaps from the branch in channel $\mathrm{A} \rightarrow \mathrm{E}$ to another branch of the same Newton trajectory which belongs to a channel to structure D. (It is an intrinsic numerical problem under the following of NTs near an extraneous singularity that the calculated series of points can leap.) The dashed circle marks the approximate place of the VRI point, which is crossed by the singular NT. There the marked NT bifurcates. One branch goes to D and another branch goes to $\mathrm{E}$. 

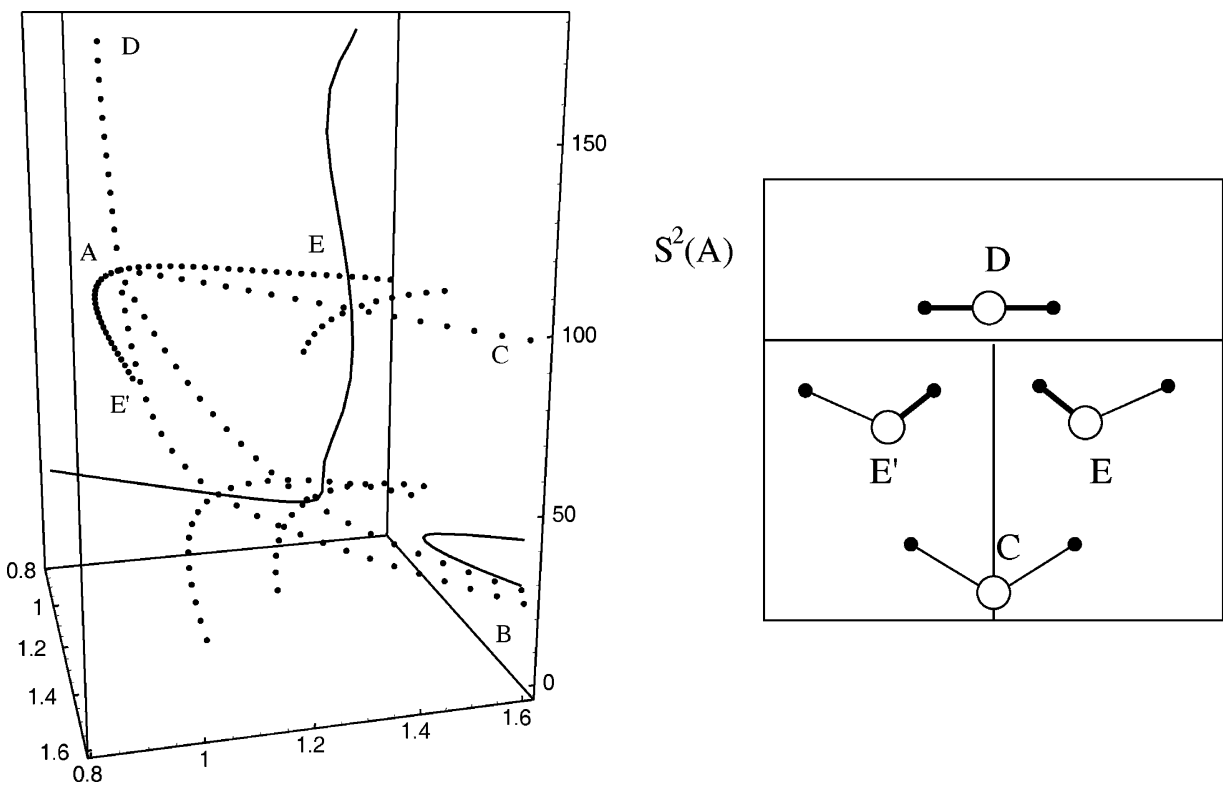

Fig. 16. Left: Newton trajectories (bullets) in configuration space of the water molecule. The full line is the symmetric VRI manifold [21]. Right: section of $S^{2}$ (A) of configurations to which the corresponding Newton trajectories lead.

The family of NTs shows the existence of a nonsymmetric VRI manifold. It belongs to the singular directions which separate the Newton channels to D and to $\mathrm{E}$, or $\mathrm{E}^{\prime}$. These directions are depicted in a schematic representation in Fig. 16 right as a horizontal line. The nonsymmetric VRI manifold was not reported in the former Ref. [21].

\subsection{The last example}

It is again $\mathrm{H}_{2} \mathrm{CO}$, see Fig. 8 above. Now we treat the 2D section of $S^{5}$ which forms all possibilities for NTs from the global minimum of formaldehyde (Fig. 18). The search directions of the NTs for the restricted case of two dimensions are the changes of the angles $\angle(\mathrm{HCO})$ and the dihedral angle. The end points of the NTs are StPs with numbering of Ref. [5]. $\psi$ axis is the part of the dihedral angle

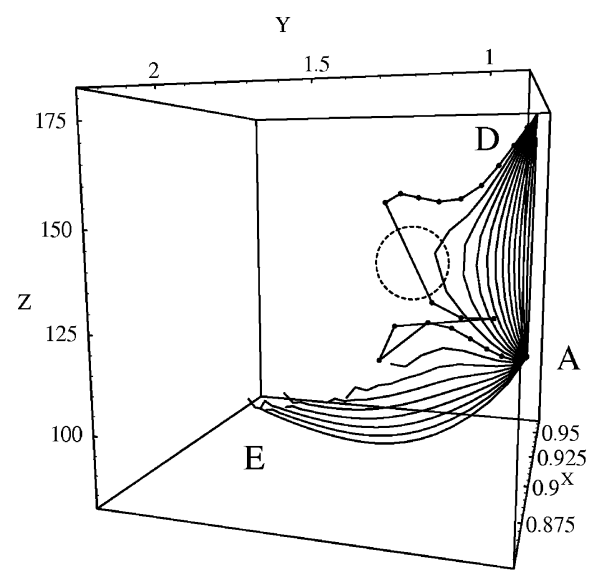

of the search direction, $\varphi$ axis is the ratio of $\angle\left(\mathrm{H}_{\alpha} \mathrm{CO}\right)$ to $\angle\left(\mathrm{H}_{\beta} \mathrm{CO}\right)$. It holds for the parts of the search direction: $\angle(\mathrm{HCOH})=\sin (\psi), \angle\left(\mathrm{H}_{\alpha} \mathrm{CO}\right)=\cos (\psi) \cos (\varphi)$ and $\angle\left(\mathrm{H}_{\beta}\right.$ $\mathrm{CO})=\cos (\psi) \sin (\varphi)$. On the $\varphi$ axis the part of the change of the dihedral angle is zero, i.e., the $C_{2}$ symmetry is hold, while on $\varphi=1.25 \pi$ (in Fig. 18 there the $\psi$ axis is depicted), the search direction is fixed to the angle symmetry $C_{2}$ (thus, the direction of the gradient). The calculated NT for $(\varphi, \psi)=(1.25 \pi, 0)$ is already reported, see Fig. 8 . Only this one NT (with respect to $S^{2} \subset S^{5}$ ) leads to StP of index 3, $\mathscr{T}_{1}$, at the cross of the axes. The region $\varphi<1.25 \pi$ and $\psi>0$ is part of the Newton channel leading from global minimum to the saddle of index $1, \mathscr{F}_{2}$. To $\psi<0$ belongs a channel which leads to the opto-isomer of $\mathscr{F}_{2}$. Every NT belonging to this $2 \mathrm{D}$ region with $\psi>0$ leads to $\mathscr{F}_{2}$. The NTs in the channel do not have singularities. NTs belonging to the 1D region of the search direction $\psi=0$ and $\varphi<1.25$

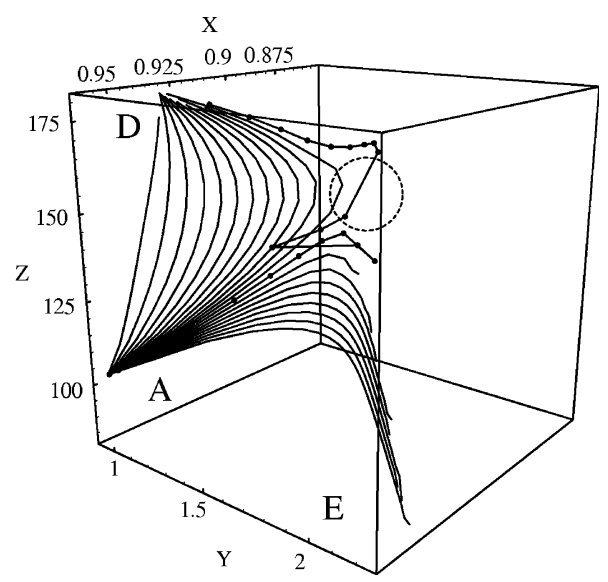

Fig. 17. A family of Newton trajectories from global minimum (A) of the MP2/ 6-31G**-PES of water. The family bifurcates into trajectories to dissociation $\mathrm{OH}+\mathrm{H}(\mathrm{E})$ and to linear SP H-O-H (D). The dashed circle marks the region of the VRI point. 


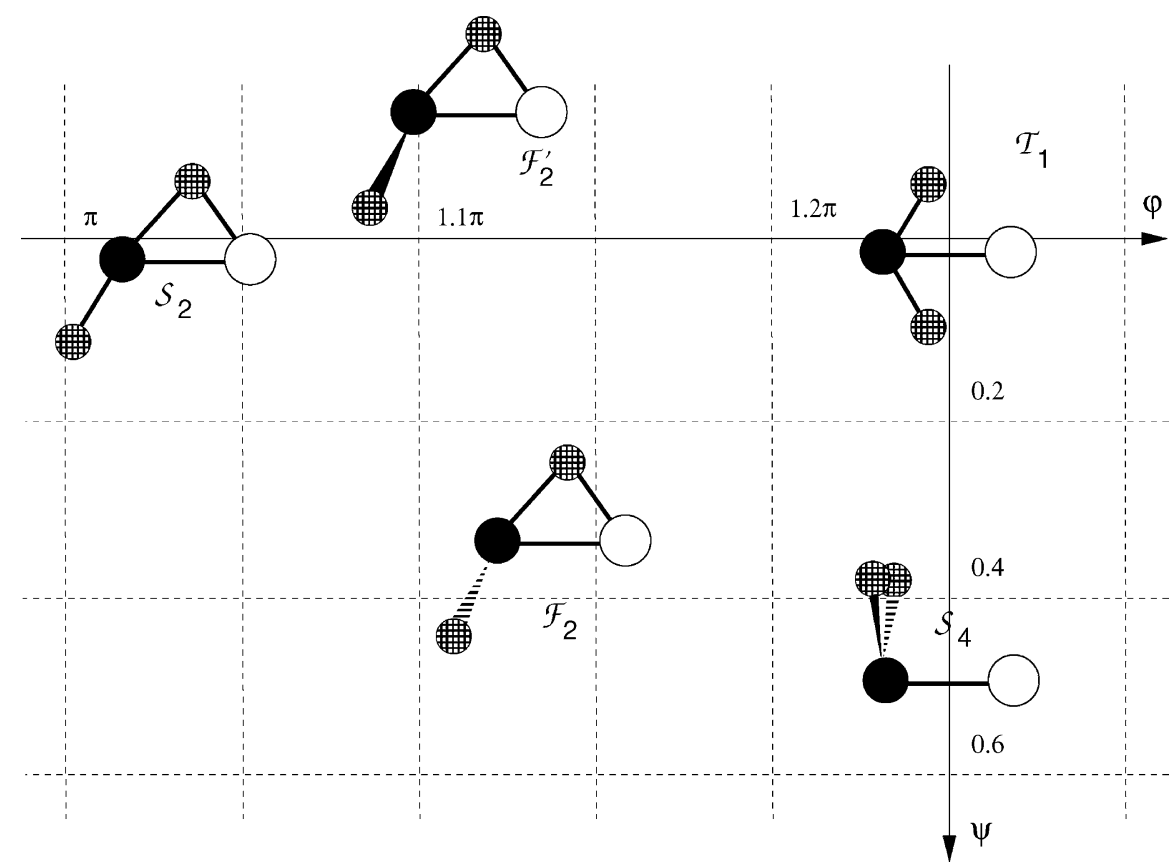

Fig. 18. Section of a Newton channel on $\mathrm{H}_{2} \mathrm{CO}$ seen from the global minimum. Structures are the stationary points which are found if the NTs are followed along the given search directions $r$ in the sectional coordinate system. It holds $r=(0,0,0, \cos (\psi) \cos (\varphi), \cos (\psi) \sin (\varphi), \sin (\psi))$.

$\pi$ lead to the SP of index $2, \mathscr{S}_{2}$. All these trajectories meet a VRI point, from which branches start again. These branches break the $C_{2}$ symmetry to $C_{1}$ symmetry and go to $\mathscr{F}_{2}$ or $\mathscr{F}_{2}^{\prime}$.

\subsection{Remark}

There are two propositions which seem very general. One is the index theorem that the change of the index along a regular branch between two stationary points is odd. We assume that the odd number is always 1 . We do not know any example where a regular branch (a branch without VRI points) connects a minimum and an SP of index 3 . The given special NT of $\mathrm{H}_{2} \mathrm{CO}$ of Fig. 8 crosses two VRI points, and the NT indicates that by an index change of 3 . The other statement is that singular points are at least a manifold of dimension $n-2$. Up to $n=4$ it holds exactly $n-2$ for the dimension of the VRI manifold (or there is no singularity). It would be of interest to precise more deeply the propositions for the application in the analysis of PESs by Newton channels.

\section{Discussion and conclusion}

The mathematical definition of a reaction channel is a general imagination which one may have about the division of the topology of the PES into diverse regions. We discussed the topology between the curves of interest here, the NTs, and the PES. The peculiarity of our ansatz is that its mathematical part is considered not as a game with deductive reasoning and symbols, but as a part of natural science, especially of chemistry, i.e. as an experimental treatment [35]. The aim of our visualizations is to support analysis and interpretation of reaction pathways. Chemists have a long tradition in inventing and applying models for the analysis of molecular transformations taking place in a reaction. The development of new ideas, definitions, and methods for modeling an RP critically depends on visualization as an effective way to gain understanding of a problem.

For a long time the IRC was the model of choice of theoretical chemistry. It is simple, and it can be compared with a dynamical model of an RP [36]. The pre-dynamical allegory of the IRC for a chemical reaction path uses a simple imagination. The system point of the reaction moves along the negative gradient without inertia. Thus, only the forces of the PES act. These forces are the gradient [37]. (Of course, the pre-dynamical picture of the IRC only works downhill.) The general treatment of steepest descent lines are the tool to treat catchment regions [10]. It is a topological division of the PES. Catchment regions of minima are the 'passive' result of the relaxation of the molecular system.

RGF is also a pre-dynamical allegory for a chemical reaction path. But another simplification is used. We assume that along the RP a force always acts into the same direction, and the force holds the trajectory on a path where the negative gradient of the PES is in equilibrium with the acting force, cf. [24]. The imagination gives the NTs. How useful is the simplification for real processes? It is open, like it is for the IRC. The NT picture may have direct implications for the attainability of an adjacent SP if one changes the direction of the acting force, for example, if one 
jumps from excitation of one normal mode to another normal mode [38].

The Newton trajectories can be used for another division of the configuration space, namely, into reaction channels. A large size of a channel gives the possibility to turn the driving force, the gradient, into very different directions. Thus, the Newton channels are understandable as the 'active' directions for the progress of a reaction from a given minimum. It seems to be a further possibility for an application of the NTs.

If one compares a Newton channel with the idea of the MEP a problem emerges. Not all NTs can be understood as MEPs [33,29], because in most cases a part of the NTs crosses a turning point (TP) before the SP. There the energy is usually higher than the energy of the SP, and so the definition of an MEP for these NTs has failed. The equipotential surface of the SP energy is the highest level, which is permitted for an MEP. The matter is developed in another paper of these authors [29]. Cutting the Newton channel by the TP criterion and putting the TP-free NTs into the 'good' part automatically also gives the valley structure of the PES from minimum to SP [29].

\section{Acknowledgements}

The work was made possible through the financial support of the Deutsche Forschungsgemeinschaft. The authors thank Prof. D. Heidrich for stimulating discussions.

\section{Appendix A. List of model surfaces}

The Müller-Brown potential [17] is used in Figs. 1, 3, $12-14$. It is

$$
E(x, y)=\sum_{i=1}^{4} A_{i} \exp \left(a_{i}\left(x-x_{i}^{0}\right)\right)^{2}+b_{i}\left(x-x_{i}^{0}\right)\left(y-y_{i}^{0}\right)+c_{i}\left(y-y_{i}^{0}\right)^{2}
$$

with

$$
\begin{aligned}
& A=(-200,-100,-170,-15) \\
& a=(-1,-1,-6.5,0.7) \\
& b=(0,0,11,0.6) \\
& c=(-10,-10,-6.5,0.7) \\
& x^{0}=(1,0,-0.5,-1 \\
& y^{0}=(0,0.5,1.5,1) .
\end{aligned}
$$

In Fig. 4 is used the potential [13]

$$
E(x, y)=0.5\left(x y^{2}-y x^{2}-x+2 y\right)+0.033\left(x^{4}+y^{4}\right) .
$$

\section{References}

[1] P.G. Mezey, Potential Energy Hypersurfaces, Elsevier, Amsterdam, 1987.

[2] D. Heidrich (Ed.), The Reaction Path in Chemistry: Current Approaches and Perspectives, Kluwer, Dordrecht, 1995.

[3] I.H. Williams, G.M. Maggiora, J. Mol. Struct. (Theochem). 89 (1982) 365.

[4] R. Czerminski, R. Elber, J. Chem. Phys. 92 (1990) 5580.

[5] W. Quapp, M. Hirsch, O. Imig, D. Heidrich, J. Comput. Chem. 19 (1998) 1087.

[6] W. Quapp, M. Hirsch, D. Heidrich, Theoret. Chem. Acc. 100 (1998) 285.

[7] M. Hirsch, Zum Reaktionswegcharakter von Newtontrajektorien, Dissertation. (Fakultät für Chemie and Mineralogie, Universität Leipzig, Dezember 2003).

[8] K. Fukui, J. Phys. Chem. 74 (1970) 4161.

[9] A. Tachibana, K. Fukui, Theoret. Chim. Acta. 49 (1978) 321. A. Tachibana, in: E.S. Kryachko, J.L. Calais (Eds), Conceptual Trends in Quantum Chemistry, Kluwer, Dordrecht, 1994, p. 101.

[10] P.G. Mezey, J. Mol. Struct. (Theochem) 123 (1985) 171. P.G. Mezey, Int. J. Quant. Chem., Symp. 19 (1986) 93.

[11] J. Milnor, Morse Theory, Princeton University Press, 5th Printing, 1973.

[12] J. Baker, P.M.W. Gill, J. Comput. Chem. 9 (1988) 465.

[13] W. Quapp, M. Hirsch, D. Heidrich, Theoret. Chem. Acc. 112 (2004) 40

[14] J.M. Anglada, E. Besalú, J.M. Bofill, R. Crehuet, J. Comput. Chem. 22 (2001) 387. J.M. Bofill, J.M. Anglada, Theor. Chem. Acc. 105 (2001) 463. R. Crehuet, J.M. Bofill, J.M. Anglada, Theor. Chem. Acc. 107 (2002) 130.

[15] H.T. Jongen, P. Jonker, F. Twilt, Nonlinear Optimization in Finite Dimensions-Morse Theory, Chebychev Approximation, Transversality, Flows, Parametric Aspects, Kluwer, Dordrecht, 2000.

[16] W. Quapp, J. Comput. Chem. 22 (2001) 537.

[17] K. Müller, L.D. Brown, Theor. Chim. Acta. 53 (1979) 75.

[18] E.L. Allgower, K. Georg, Numerical Continuation Methods-An Introduction, Springer, Berlin, 1990.

[19] I. Diener, Globale Aspekte des kontinuierlichen Newtonverfahrens. Habilitation, Göttingen, 1991.

[20] F.H. Branin, IBM J. Res. Dev. 16 (1972) 504.

[21] M. Hirsch, W. Quapp, D. Heidrich, Phys. Chem. Chem. Phys. 1 (1999) 5291.

[22] W. Quapp, V. Melnikov, Phys. Chem. Chem. Phys. 3 (2001) 2735.

[23] W. Quapp, D. Heidrich, J. Mol. Struct. (Theochem) 585 (2002) 105.

[24] W. Kliesch, J. Comput. Chem. 22 (2001) 1801.

[25] F. Jensen, Theor. Chim. Acta. 99 (1998) 295.

[26] M. Hirsch, W. Quapp, J. Comput. Chem. 23 (2002) 887.

[27] E. Kreyszing, Differentialgeometrie, Akad. Verl. Ges. Geest and Porting, Leipzig, 1968.

[28] L. Volkmann, Graphen und Digraphen: Eine Einführung in die Graphentheorie, Springer, Wien, New York, 1991.

[29] M. Hirsch, W. Quapp, J. Math. Chem. (2004) in press.

[30] P.G. Mezey, P. Warburton, E. Jako, Z. Szekeres, J. Math. Chem. 30 (2001) 375.

[31] D.J. Wales, J.P.K. Doye, J. Chem. Phys. 119 (2003) 12409.

[32] J. Kurchan, L. Laloux, J. Phys. A 29 (1996) 1929.

[33] W. Quapp, J. Theor. Comp. Chem. 2 (2003) 385.

[34] W. Quapp, M. Hirsch, D. Heidrich, Theoret. Chem. Acc. 105 (2000) 145.

[35] V.I. Arnold, Arnold's Problems, Springer, Berlin, 2004.

[36] J. González, X. Giménez, J.M. Bofill, Phys. Chem. Chem. Phys. 4 (2002) 2921.

[37] K. Müller, Angew. Chem. 92 (1980) 1.

[38] L. Windhorn, J.S. Yeston, T. Witte, W. Fuß, M. Motzkus, D. Proch, K.-L. Kompa, J. Chem. Phys. 119 (2003) 641. 